\title{
Erratum zu: Erwachsenen- und Weiterbildung unter Pandemiebedingungen. Herausforderungen und Perspektiven
}

\author{
Kerstin Hoenig • Gabriele Molzberger
}

Online publiziert: 21. Januar 2022

(C) Der/die Autor(en) 2021

\section{Erratum zu:}

\section{ZfW 2021}

https://doi.org/10.1007/s40955-021-00197-0

Im Editorial dieser Ausgabe wurde der Name der Koautorin des Beitrags „COVID19 und Weiterbildung - Überblick zu Forschungsbefunden und Desiderate“ nicht erwähnt: Anika Denninger. Das Editorial wurde korrigiert und nochmals veröffentlicht. Wir bedauern diesen Fehler.

Der Originalbeitrag wurde korrigiert.

Open Access Dieser Artikel wird unter der Creative Commons Namensnennung 4.0 International Lizenz veröffentlicht, welche die Nutzung, Vervielfältigung, Bearbeitung, Verbreitung und Wiedergabe in jeglichem Medium und Format erlaubt, sofern Sie den/die ursprünglichen Autor(en) und die Quelle ordnungsgemäß nennen, einen Link zur Creative Commons Lizenz beifügen und angeben, ob Änderungen vorgenommen wurden.

Die in diesem Artikel enthaltenen Bilder und sonstiges Drittmaterial unterliegen ebenfalls der genannten Creative Commons Lizenz, sofern sich aus der Abbildungslegende nichts anderes ergibt. Sofern das betreffende Material nicht unter der genannten Creative Commons Lizenz steht und die betreffende Handlung

Die Online-Version des Originalartikels ist unter https://doi.org/10.1007/s40955-021-00197-0 zu finden.

Dr. Kerstin Hoenig $(\bowtie)$

Deutsches Institut für Erwachsenenbildung - Leibniz-Zentrum für Lebenslanges Lernen, Bonn, Deutschland

E-Mail: hoenig@die-bonn.de

Prof. Dr. Gabriele Molzberger

Bergische Universität Wuppertal, Wuppertal, Deutschland

E-Mail: molzberger@uni-wuppertal.de 
nicht nach gesetzlichen Vorschriften erlaubt ist, ist für die oben aufgeführten Weiterverwendungen des Materials die Einwilligung des jeweiligen Rechteinhabers einzuholen.

Weitere Details zur Lizenz entnehmen Sie bitte der Lizenzinformation auf http://creativecommons.org/ licenses/by/4.0/deed.de. 